\title{
ANALYSIS OF INTERFACIAL AND MASS TRANSFER EFFECTS ON FORCED CONVECTION IN GAS-LIQUID ANNULAR TWO- PHASE FLOW
}

\author{
E. Nogueira \\ B. D. Dantas \\ Research and Development Institute IP\&D- \\ UNIVAP. Av. Shishima Hifumi, 2911 \\ São José dos Campos - SP, 12244-000, Brazil \\ E-mail: elcio@univap.br \\ E-mail: lucilia@univap.br \\ R. M. Cotta \\ DEM/EE \& PEM/COPPE/UFRJ \\ Cidade Universitária, Cx. Postal 68503 \\ Rio de Janeiro, RJ, 21945-970 \\ E-mail: cotta@serv.com.ufrj.br
}

\begin{abstract}
In a gas-liquid annular two-phase flow one of the main factors influencing the determination of heat transfer rates is the average thickness of the liquid film. A model to accurately represent the heat transfer in such situations has to be able of determining the average liquid film thickness to within a reasonable accuracy. A typical physical aspect in gas-liquid annular flows is the appearance of interface waves, which affect heat, mass and momentum transfers. Existing models implicitly consider the wave effects in the momentum transfer by an empirical correlation for the interfacial friction factor. However, this procedure does not point out the difference between interface waves and the natural turbulent effects of the system. In the present work, the wave and mass transfer effects in the theoretical estimation of average liquid film thickness are analyzed, in comparison to a model that does not explicitly include these effects, as applied to the prediction of heat transfer rates in a thermally developing flow situation.
\end{abstract}

Keywords: gas-liquid annular flow, interface wave effects, heat and mass transfer, analytical methods.

\section{INTRODUCTION}

Gas-liquid annular two-phase flow has practical applications in several industrial processes and for this reason a rather large number of research works on heat transfer in two-phase flows have given emphasis to the annular flow regime. The more analytically oriented contributions, with few exceptions such as in (Suzuki et al., 1983 and Dobran, 1985), are essentially devoted to the analysis of the heat transfer process in the liquid film only, thus, in most of the cases neglecting the influence of the gas core and the complex interfacial phenomena. Also, not so many previous works seem to be concerned with the thermal entry region analysis for a gas-liquid annular flow.

The majority of the contributions on heat transfer in annular two-phase flows is related to the correlation of experimental data. Although satisfactory in different practical situations, such correlations cannot possibly recover and represent all such flow characteristics that affect the heat transfer process, for instance, interface waves, liquid entrainment, condensation-evaporation (Hewitt, 1993 and Silvestri, 1964), and more elaborate modeling is then required (Suzuki et al., 1983, Dobran, 1985 and Nogueira, 1993).

The vertical gas-liquid annular flow has been extensively studied and models have been proposed to calculate the pressure gradient, mean film thickness and other characteristics of the flow regime. For example, just to illustrate some recent contributions, air-water up ward annular flow experiments were conducted in a $10.8 \mathrm{~m}$ long tube of $31.8 \mathrm{~mm}$ internal diameter by Wolf et al. (2001). They reported measurements of pressure gradient, film thickness, wall shear stress, film flow rate, disturbance wave velocity and frequency, over a range of gas-liquid flow rates. Also quite recently, the churn flow regime in vertical gas liquid flow was investigated by Barbosa Jr. et al. (2001). They described a series of experiments using a test section with a specially constructed transparent liquid inlet. High-speed video recordings showed clearly the process of wave formation and analysis of the recordings gave data on wave frequencies and typical velocities.

The present work is concerned with the analytical solution of a hydrodynamically developed and thermally developing flow model, for two-phase gas-liquid annular flow, heat and mass transfer. The wave effects in the theoretical estimation of the average liquid film thickness are analyzed in relation to a model that does not explicitly include these effects and does not consider their influence on the overall heat and mass transfer process. The partial differential system is analytically solved through the integral transform method, and numerical results are critically compared against available experimental findings.

In Nogueira et al., (1995) a few comparisons were performed considering the theoretical model for the twophase flow with turbulence, entrainment and interface waves effects, against available experimental results at different Reynolds numbers and gas flow rates. The three major quantities were then analyzed, respectively, average liquid film thickness $(\bar{m})$, the pressure gradient $(\mathrm{dp} / \mathrm{dz})$ and the local velocity profiles $\left(\mathrm{u}_{\mathrm{k}}(\mathrm{r})\right)$, and critically compared with 
measurements from Zabaras et al. (1986) for $\bar{m}$ and $\mathrm{dp} / \mathrm{dz}$ and measurements from Jensen, (1987) and Gill et al., $(1963,1964)$ for $u_{k}(r)$. The results presented by Nogueira et al. (1995) demonstrated that the alternative procedure introduced was relatively consistent and precise, without requiring the use of involved correlations. A comparison was also performed against the theoretical model proposed by Suzuki et al. (1983), which incorporated the same treatment of the interface waves adopted by Nogueira et al. (1995), but did not include a corrective procedure for liquid entrainment effects. For situations of low entrainment rates, a good agreement was achieved between the two theoretical predictions. Although the diffusivity model associated with the interface waves did not significantly alter the results for the liquid film thickness and pressure gradient, it was of major relevance in the evaluation of the temperature field, as shown by Nogueira et al. (1995) within their heat transfer analysis. Results were then presented to confirm this behavior, in terms of heat transfer coefficient along the channel length, for different values of gas flow rates and Reynolds number. They observed that the interface waves effect was more pronounced for the larger values of Reynolds number associated with the liquid film. The heat transfer enhancement effect was quite noticeable and the proposed model (Nogueira et al., 1995) corrected the theoretical prediction towards an improved agreement with the experimental results obtained by Hagiwara et al. (1982).

In this work, this modeling effort is progressed, by again taking the theoretical flow model of Nogueira et al. (1995), which is now utilized to analyze the combined effects of heat and mass transfer.

\section{THEORETICAL ANALYSIS}

A differential model is here proposed for annular two-phase flow and heat transfer, departing from the hypothesis of a fully developed velocity profile on both gas and liquid phases, and then a thermally developing region is considered and analyzed. The following two sections deal separately with the flow and heat transfer problems, for the sake of clarity.

\section{Flow Problem}

The momentum diffusion process is described by three components, namely, the viscous component, the turbulence effect itself and a term that accounts for the waves at the phases interface. The turbulent diffusivity is obtained from extensions to classical algebraic models for single-phase flows (Deissler, 1950, Kays and Crawford, 1980), as described in the following. The interface waves effect is modeled through the expressions proposed by Suzuki et al. (1983).

For the sake of generality, the geometry under consideration allows for both a circular tube and parallel-plates channel configuration, besides including the possibility of a slight duct inclination from the vertical position, provided a symmetric flow condition can still be, at least approximately, employed in the mathematical formulation.

Nogueira et al., $(1990,1995)$ have demonstrated that the velocity profiles in both regions of the flow are obtained by the expressions:

$$
\begin{aligned}
& u_{1}(R)=\frac{P_{2} r_{2}^{2}}{(n+1) \mu_{2}}\left\{\hat{\mu} \hat{P} \int_{R}^{\delta} R^{\prime} d R^{\prime}+\right. \\
& \left.\int_{\delta}^{1}\left[R^{\prime}+\frac{(\hat{P}-1) \delta^{n+1}}{R^{\prime n}}\right]\right\} \frac{d R^{\prime}}{E_{m 2}\left(R^{\prime}\right)} \\
& u_{2}(R)=\frac{P_{2} r_{2}^{2}}{(n+1) \mu_{2}}+\left\{\int _ { R } ^ { 1 } \left[R^{\prime}+\right.\right. \\
& \left.\left.\frac{(\hat{P}-1) \delta^{n+1}}{R^{\prime n}}\right]\right\} \frac{d R^{\prime}}{E_{m 2}\left(R^{\prime}\right)}
\end{aligned}
$$

with the following related parameters

$$
\begin{aligned}
& R=\frac{r}{r_{2}}, \quad \delta=\frac{r_{1}}{r_{2}}, \quad \hat{P}=\frac{P_{1}}{P_{2}}, \quad \hat{\mu}=\frac{\mu_{1}}{\mu_{2}}, \\
& P_{k}=-\left[\frac{d p}{d z} \pm \rho_{k} g_{\alpha}\right], \quad g_{\alpha}=|g| \sin \alpha \\
& E_{m k}\left(R, u_{k}\right)=1+\frac{\varepsilon_{m k}\left(R, u_{k}\right)}{v_{k}}+\frac{\varepsilon_{o k}(R)}{v_{k}}
\end{aligned}
$$

The three components of the overall diffusivity are identified within the expression above, eq.(1.i). Also, the index $\mathrm{k}$ stands for the phase in consideration $(k=1$, for the gas core; $k=2$, for the liquid film), while $\mathrm{n}$ stands for the geometry ( $n=1$, for circular tube and $n=0$, for parallel plates).

Then, the shear stresses distributions may be computed from:

$$
\begin{array}{ll}
\tau_{1}(R)=\frac{r_{2} P_{1} R}{(n+1)} & 0 \leq R \leq \delta \\
\tau_{2}(R)=\frac{r_{2} P_{2}}{(n+1)}\left[R+\delta^{n+1} \frac{(\hat{P}-1)}{R^{n}}\right] \delta \leq R \leq 1
\end{array}
$$

The average flow velocity is obtained by

$$
\begin{aligned}
& \bar{u}=\frac{P_{2} r_{2}^{2}}{(n+1) \mu_{2}}\left\{\hat{\mu} \hat{P} \int_{0}^{\delta} \frac{R^{n+2}}{E_{m 1}(R)} d R+\right. \\
& \left.\int_{\delta}^{1} R^{n+1}\left[R+\frac{(\hat{P}-1) \delta^{n+1}}{R^{n}}\right] \frac{d R}{E_{m 2}(R)}\right\}
\end{aligned}
$$

A dimensionless velocity is defined as

$$
U(R)=\frac{u_{k}(r)}{C \bar{u}}
$$


where $\mathrm{C}$ is a parameter introduced to allow for direct comparisons against previous works.

The algebraic turbulence models adopted are obtained from the related literature (Deissler, 1950, Kays and Crawford, 1980), and given by:

$$
\begin{aligned}
& \frac{\varepsilon_{m k}}{v_{k}}=l^{2} u_{k}^{+}(R) y_{k}^{+}\left[1-\exp \left(l^{2} u_{k}^{+}(R) y_{k}^{+}\right)\right], \\
& \text {for } y_{k}^{+} \leq 20, R_{k-1} \leq R \leq R_{k}, \quad k=1,2
\end{aligned}
$$

with $l^{2}=0.0154$, as proposed by Deissler (1950), and

$$
\begin{aligned}
& \frac{\varepsilon_{m k}}{v_{k}}=\frac{k_{1}^{\prime}}{6} y_{k}^{+}\left[1+R\left(\frac{r_{2}}{r_{1}}\right)\right]\left[1+2 R^{2}\left(\frac{r_{2}}{r_{k}}\right)^{2}\right], \\
& \text { for } y_{k}^{+} \leq 20, R_{k-1} \leq R \leq R_{k}, \quad k=1,2
\end{aligned}
$$

with $k_{1}^{\prime}=0.4$, as obtained by Reichardt (Kays and Crawford, 1980), and related quantities are given by

$$
\begin{aligned}
& y_{k}^{+}=\left(\delta^{2-k}-R\right) r_{2} \frac{\sqrt{\tau_{k}\left(R_{k}\right) / \rho_{k}}}{v_{k}} \\
& R_{k-1} \leq R \leq R_{k}, \quad k=1,2 \\
& u_{k}^{+}=\frac{u_{\boldsymbol{k}}(R)-u_{\boldsymbol{k}}\left(R_{k}\right)}{\sqrt{\tau_{k}\left(R_{k}\right) / \rho_{k}}}, R_{k-1} \leq R \leq R_{k}, \quad k=1,2
\end{aligned}
$$

With respect to the interface waves effects, the procedure employed to determine an effective average diffusivity, $\varepsilon_{o k}$, was adapted from the model presented by Suzuki et al. (1983), in the general form:

$$
\frac{\varepsilon_{o k}}{v_{k}}=\frac{c_{o} L^{2} F}{v_{k}} \quad k=1,2
$$

where $F$ represents the average number of waves passing, by unit of time, through the channel cross section, $L$ is the average wave height and $c_{o}$ is an adjustment constant adopted in the original procedure. The above expression is then utilized within a region in the neighborhood of the interface, defined by:

$$
\begin{array}{ll}
\frac{\varepsilon_{o 2}}{v_{2}}=0 & \text { for } 0 \leq y_{2}^{+} \leq 5 ; \\
\frac{\varepsilon_{o 2}}{v_{2}}=\frac{c_{o} L^{2} F}{v_{2}}, & \text { for } y_{2}^{+}>5 \\
\frac{\varepsilon_{o 1}}{v_{1}}=\frac{c_{o} L^{2} F}{v_{1}} \text { and } \frac{\varepsilon_{m 1}}{v_{1}}=0 \quad \text { for } y_{1}^{+}<y_{1}^{+}, \\
\frac{\varepsilon_{o 1}}{v_{1}}=0 & \text { for } y_{1}^{+}>y_{1}^{+\bullet}
\end{array}
$$

where $y_{1}^{+\bullet}$ is determined from the transcendental equation

$$
\frac{\varepsilon_{m 1}}{v_{1}}=\frac{\varepsilon_{o 1}}{v_{1}}
$$

In the annular two-phase flow literature, $W_{L}, W_{L F}$, and $W_{L E}$ are the mass flow rates associated, respectively, with the total liquid, fraction of liquid in the film and the fraction dispersed within the gas core (entrainment). Therefore,

$$
W_{L}=W_{L F}+W_{L E}
$$

and a characteristic Reynolds number may be defined as

$$
R e_{L S}=\frac{4 W_{L}}{\rho \mu_{L}}
$$

The scheme for computing the velocity profiles and related quantities is described in detail in (Nogueira, 1993 and Nogueira et al., 1990), being a similar approach to the one proposed by Walley (1987).

Nogueira et al. (1995) also demonstrated that consideration of the interface waves diffusivity term increases the magnitude of the predicted liquid film thickness, with respect to the case without including such effect, and, in most cases, with slight overestimates with respect to the experimental findings. It was then clear that the flow predictions were not so markedly affected by the interface waves correction factor, as they were by thodijquid entrainment correction. It was noticed, however, that within the model, the interface waves effect was in any case approximately accounted for due to the allowed discontinuity at the interface shear stress, evaluated from empirical correlations. Therefore, the turbulent diffusivity is always affected somehow by the presence of the interface waves, which act as a wall roughness effect on the gas flow field.

\section{Heat Transfer Problem}

We consider heat transfer by forced convection between the duct wall and the fluid streams, under the hypothesis of hydrodynamically developed and thermally developing flow. In addition to the annular two-phase flow hypothesis, constant physical properties have been considered. Viscous dissipation, axial conduction, and natural convection effects are neglected.

The general dimensionless formulation of the problem is established through the following equations of energy and their interface and boundary conditions:

$$
\begin{gathered}
\left(\frac{l_{0}}{l_{n}}\right)^{2} C\left(1+\beta_{k}\right) \frac{\rho_{k} C_{k}}{\bar{\rho} \bar{C}} R^{n} U_{k}(R) \frac{\partial \theta_{k}(R, Z)}{\partial Z}= \\
\frac{\partial}{\partial R}\left[\left(1+\beta_{k}\right) R^{n} \frac{k_{k}}{\bar{k}} E_{h k}(R) \frac{\partial \theta_{k}(R, Z)}{\partial R}\right] \\
Z>0, R_{k-1} \leq R \leq R_{k}, \quad k=1,2 \\
\theta_{k}(R, 0)=F_{k}(R), \quad R_{k-1} \leq R \leq R_{k}, \quad k=1,2
\end{gathered}
$$


$\frac{\partial \theta_{1}(R, Z)}{\partial R}=0, \quad Z>0, R=0$

$\theta_{1}(R, Z)=\theta_{2}(R, Z), Z>0, R=\delta$

$\left(1+\beta_{1}\right) R^{n} \frac{k_{1}}{\bar{k}} E_{h 1}(R) \frac{\partial \theta_{1}(R, Z)}{\partial R}=$

$\left(1+\beta_{2}\right) R^{n} \frac{k_{2}}{\bar{k}} E_{h 2}(R) \frac{\partial \theta_{2}(R, Z)}{\partial R}, Z>0, R=\delta$

$(8 \mathrm{e})$

$$
\begin{aligned}
& \frac{\alpha^{\bullet} l_{0}}{\bar{k}}\left[\theta_{2}(R, Z)+\frac{T^{\bullet}}{\Delta T}\right]+\quad Z>0, R=1 \\
& \beta \cdot \frac{k_{2}}{\bar{k}} E_{h 2}(R) \frac{\partial \theta_{2}(R, Z)}{\partial R}=\Phi(Z),
\end{aligned}
$$

The various dimensionless groups appearing above are defined as:

$$
\begin{aligned}
& Z=\frac{\bar{\alpha} z}{\bar{u} l_{n}^{2}}, \quad \theta_{k}(R, Z)=\frac{T_{k}(r, z)-T^{*}}{\Delta T}, \\
& F_{k}(R)=\frac{f_{k}(r)-T^{*}}{\Delta T}, \quad \Phi(Z)=\frac{\phi(z) l_{0}}{\bar{k} \Delta T}
\end{aligned}
$$

d)

$$
\begin{aligned}
& E_{h k}\left(R, u_{k}\right)=1+\operatorname{Pr}_{k} \frac{\varepsilon_{h k}(R)}{v_{k}}= \\
& 1+\frac{\operatorname{Pr}_{k}}{\operatorname{Pr}_{k t}}\left(\frac{\varepsilon_{m k}\left(R, u_{k}\right)}{v_{k}}+\frac{\varepsilon_{o k}(R)}{v_{k}}\right) \\
& l_{0}=r_{2}, \quad l_{n}=2 r_{2}, \quad \bar{\alpha}=\frac{\bar{k}}{\bar{\rho} \bar{C}}, \\
& \bar{k}=\delta^{n+1} k_{1}+\left(1-\delta^{n+1}\right) k_{2}, \\
& \bar{\rho}=\delta^{n+1} \rho_{1}+\left(1-\delta^{n+1}\right) \rho_{2}, \\
& \bar{C}=\delta^{n+1} C_{1}+\left(1-\delta^{n+1}\right) C_{2} .
\end{aligned}
$$

where $P r_{k}$ and $P r_{k t}$ are the Prandtl numbers that relate the diffusivities of heat and momentum, respectively, that incorporate both effects of eddies and waves, $Z$ is the axial coordinate, $\theta$ is the dimensionless temperature, $F(R)$ is the inlet temperature, $\Phi(Z)$ is the prescribed boundary source function, $C$ is the constant employed in the dimensionless velocity expression, $l_{0}$ is the reference length $\left(=r_{2}\right), l_{n}$ is another reference length $\left(=2 r_{2}\right), r_{0}$ is the channel centerline position $\left(r_{0}=0\right), r_{1}$ is the interface position, $r_{2}$ is the channel wall position, $E_{h k}(R)$ is the dimensionless thermal diffusivity of phase $k, E_{t k}(R)$ is the dimensionless momentum diffusivity of phase $k$, $\alpha_{k}$ is the thermal diffusivity of the phase $k, \delta$ is the dimensionless interface position $\left(=r_{1} / r_{2}\right), \varepsilon_{m \mathrm{k}}$ is the turbulent diffusivity of phase $k, \varepsilon_{h \mathrm{k}}$ is the eddy diffusivity of phase $k, \mu_{k}$ is the viscosity of phase $k$, $\hat{\mu}$ is the viscosities ratio $\left(=\mu_{2} / \mu_{1}\right), \rho_{k}$ is the density of the phase $k, \phi(z)$ is the boundary condition source term, $v_{k}$ is the kinematic viscosity of phase $k, \tau_{k}$ is the shear stress associated to phase $k, \tau$ is the interface shear stress, and finally $\alpha^{\circ}$ and $\beta^{\circ}$ are prescribed coefficients that recover the wall boundary conditions type, $i$. e., prescribed temperature, prescribed heat flux or convective type.

The exact formal solution of the dimensionless problem defined above is obtained through application of the integral transform technique (Mikhailov and Özisik, 1984 and Cotta, 1993), as:

$$
\begin{aligned}
& \theta_{k}(R, Z)=\sum_{i=1}^{\infty} \frac{\Psi_{k}\left(\mu_{i}, R\right)}{N_{i}} e^{-\lambda_{i}^{2} Z} \\
& {\left[\bar{f}_{i}+\left(\frac{l_{n}}{l_{0}}\right)^{2} \frac{1}{C} \int_{0}^{Z} \bar{g}_{i}\left(Z^{\prime}\right) e^{\lambda_{i}^{2} Z^{\prime}} d Z^{\prime}\right]}
\end{aligned}
$$

where $\Psi_{k}\left(\mu_{i}, R\right) \equiv \Psi_{k i}(R), \quad \mu_{i}, N_{i} \equiv N\left(\mu_{i}\right), \quad \bar{f}_{i} \equiv \bar{f}\left(\mu_{i}\right)$, $\bar{g}_{i}(Z) \equiv \bar{g}\left(\mu_{\mathrm{i}}, Z\right) \quad$ and $\quad W_{k}(R) \quad$ are eigenfunctions, eigenvalues, norms, transformed inlet conditions, transformed source terms and the weighting function, respectively, given by

$$
\begin{aligned}
& N\left(\mu_{i}\right)=\sum_{k=1}^{2} \int_{R_{k-1}}^{R_{k}} W_{k}(R) \Psi_{k}^{2}\left(\mu_{i}, R\right) d R \\
& \bar{f}\left(\mu_{i}\right)=\sum_{k=1}^{2} \int_{k_{k-1}}^{R_{K}}(R) \Psi_{k}\left(\mu_{i}, R\right) F_{k}(R) d R \\
& \bar{g}\left(\mu_{i}, Z\right)=\Phi^{\bullet}(Z)\left[\frac{\Psi_{2}\left(\mu_{i}, 1\right)-K_{2}(1) \Psi_{2}^{\prime}\left(\mu_{i}, 1\right)}{\alpha^{\prime}+\beta^{*}}\right] \\
& \alpha^{\prime}=\frac{\alpha^{\bullet} l_{o}}{\bar{k}} \\
& \Phi^{\bullet}(Z)=\Phi(Z)-\alpha^{\prime} \frac{T^{*}}{\Delta T} \\
& W_{k}(R)=\left(1+\beta_{k}\right) \frac{\rho_{k} C_{k}}{\bar{\rho} \bar{C}} R^{n} U_{k}(R) \\
& \lambda_{i}^{2}=\frac{1}{C}\left(\frac{l_{n}}{l_{o}}\right)^{2} \mu_{i}^{2}
\end{aligned}
$$

The associated eigenvalue problem is proposed and solved by Nogueira et al.(1995).

Due to a possible slow convergence of the related infinite series defined through Eq. (10a), especially for the case of a prescribed heat flux boundary condition $\left(\alpha^{\bullet}=0\right.$, $\left.\beta^{\bullet}=1\right)$, it is convenient to rewrite the solution of our problem through an analytical "Splitting-up" procedure (Mikhailov and Özisik, 1984), in the form:

$$
\theta_{k}(R, Z)=\bar{\theta}(Z)+\theta_{k Z}(R, Z)+\theta_{k 0}(R)
$$

where the dimensionless average temperature, $\bar{\theta}(Z)$, is defined as 


$$
\bar{\theta}(Z)=\frac{\frac{2 Z}{C}+\sum_{k=1}^{2} \int_{R_{k-1}}^{R_{k}} W_{k}(R) F_{k}(R) d R}{\sum_{k=1}^{2} \int_{R_{k-1}}^{R_{k}} W_{k}(R) d R}
$$

The solution of the homogeneous problem within the entrance region, $\theta_{k Z}(R, Z)$, is obtained as

$$
\theta_{k Z}(R, Z)=\sum_{i=1}^{\infty} \frac{\psi_{k i}(R)}{N_{i}} e^{-\lambda_{i}^{2} Z}\left[\bar{f}_{i}-\frac{\bar{g}_{i}(Z)}{\mu_{i}^{2}}\right](14)
$$

Finally, the component $\theta_{k o}(R)$ is analytically determined to yield (Nogueira, 1993):

$$
\theta_{k o}=\Phi(Z)\left[H_{k}^{\prime}(R)-\frac{\sum_{k=1}^{2} \int_{R_{k-1}}^{R_{k}} W_{k}(R) H_{k}^{\prime}(R) d R}{\sum_{k=1}^{2} \int_{R_{k-1}}^{R_{k}} W_{k}(R) d R}\right]
$$

where

$$
H_{1}^{\prime}(R)=\int_{0}^{R} \frac{H_{1}\left(R^{\prime}\right)}{K_{1}\left(R^{\prime}\right)} d R^{\prime}
$$

b)

$$
H_{2}^{\prime}(R)=H_{1}^{\prime}(R)+\int_{0}^{R} \frac{H_{1}\left(\delta^{\prime}\right)+H_{2}\left(R^{\prime}\right)}{K_{2}\left(R^{\prime}\right)} d R^{\prime}
$$

c)

$$
H_{k}=\frac{\int_{R_{k-1}}^{R_{k}} W_{k}\left(R^{\prime}\right) d R^{\prime}}{\sum_{k=1}^{2} \int_{R_{k-1}}^{R_{k}} W_{k}(R) d R}, \quad k=1,2
$$

d)

From the temperature distribution, Eq. (12), the heat transfer coefficient at the channel wall can be evaluated, for instance, in the case of a prescribed wall heat flux condition, as:

$$
h(z)=\frac{-\left.k_{2} \frac{\partial T_{2}}{\partial r}\right|_{r=r_{2}}}{\bar{T}(z)-T_{2}\left(r_{2}, z\right)}=\frac{\phi(z)}{T_{2}\left(r_{2}, z\right)-\bar{T}(z)}
$$

The heat transfer process in annular twophase flows is very complex, because it is affected by several factors, such as: liquid and gas flow rates, liquid film thickness, liquid entrainment in the core and interfacial waves effects. Another important factor, also of interest here, is the mass transfer process. Previous works have pointed out the concern with mass transfer in annular two-phase flows (Suzuki at al., 1983, Hewitt and Hall-Taylor, 1970, Collier, 1972), but a very definitive analysis of its influence on heat transfer rates determination is not apparently available. Actually, for a high evaporation latent heat, this factor may be significant in the heat transfer process when the wall heat fluxes are high enough. In this work, the dimensionless parameter, $\beta_{k}$, that expresses the mass transfer effect on the energy balance, Eqs. (8a) and (8e), due to the liquid film evaporation, is modeled through the expression proposed by Suzuki at al. (1983), in a much similar way. A detailed mathematical model is presented in Nogueira (1993), but here it suffices to present the expression employed for this parameter:

$$
\beta_{1}=\beta_{g}=\frac{H_{L} M_{v}}{M_{g} V_{v} C_{g} P T_{m}}
$$

where $H_{L}$ is the evaporation latent heat, $M_{v}$ is the molecular mass of vapor, $M_{g}$ is the molecular mass of gas, $V_{v}$ is the specific volume of vapor, $C_{g}$ is the specific heat, $P$ is total pressure and $T_{m}$ is the bulk temperature.

In this analysis, we also assume that in the evaporation process the heat flux is sufficiently low and, consequently, the maximum temperature difference between the wall and the duct center duct is at most around $20^{\circ} \mathrm{C}$ (Hagiwara et al., 1982). Also, it is assumed that the parameter $\beta_{l}$, depends on the bulk temperature, and is thus independent of the local temperature. In the present model, the parameter $\beta_{l}$, affects only the interface, as shown through Eq. (8e).

For the determination of $\beta_{l}$, it is necessary to obtain the value for the bulk temperature at a given position $Z$, which requires an iterative numerical procedure. Here, just for the sake of observing the influence of this parameter on the heat transfer process, the temperature was assumed to be uniform at the entry temperature value and the numerical value of $\beta_{l}$ was then obtained as a fixed value, that due to the small temperature variation was considered to be constant along the axial direction.

\section{RESULTS AND DISCUSSION}

The solution of the heat transfer problem here proposed depends essentially on the determination of the velocity field and on the accurate evaluation of eigenvalues and eigenfunctions from problem (12). Once the velocity field determination has been verified in previous investigations (Nogueira et al, 1990, and Nogueira et al., 1995), and the numerical solution of the associated SturmLiouville system has been extensively validated (Cotta \& Nogueira, 1988, Cotta, 1993, Nogueira et al., 1996), we are left with the task of examining the adequacy of the constructed heat transfer modeling for the thermal entrance region.

We first examine the influence of the mass transfer effect, due to film evaporation, on the heat transfer coefficient along the thermal entrance region. Figure 1 illustrates such effect for one representative situation. It can be observed that the mass transfer effect has little influence in improving the heat transfer modeling, but this is due 
partially to the assumptions taken within this specific simulation (low heat flux and uniform temperature), and not entirely due to the adopted model. At the present stage, it was considered sufficiently relevant to observe the phenomena, and confirm its physical consistency in increasing the heat transfer coefficients.

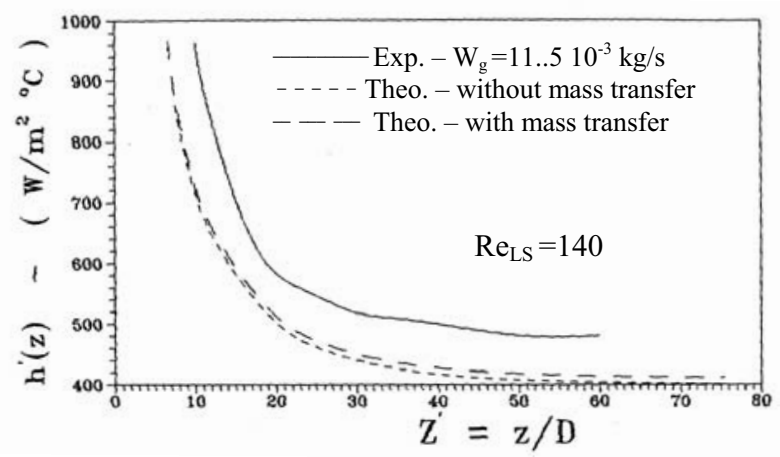

Figure 1. Comparison of theoretical (with and without mass transfer) and experimental results presented by Hagiawara et al. (1982) for the heat transfer coefficient.

We now concentrate on a wider range of governing parameters for analysis of the heat transfer coefficient simulation, also considering previous modeling efforts (Suzuki et al., 1983). Figure 2 illustrates a comparison of the present theoretical model for two-phase flow with interface waves and mass transfer effects against available experimental results obtained by Suzuki et al., (1983).

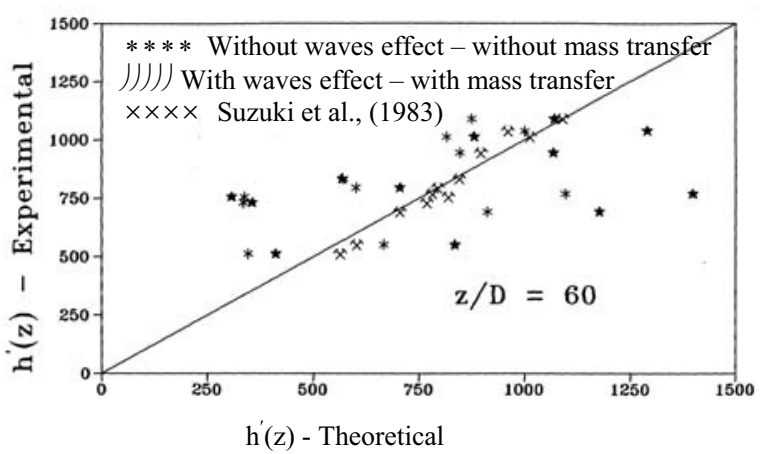

Figure 2. Comparison of theoretical (with and without waves effect) and experimental results for the heat transfer coefficient

It should be observed that the results obtained by Suzuki et al. (1983) present a less marked dispersion in comparison to those achieved in the present work. The differences observed can be justified in some aspects. First, in the Suzuki et al. (1983) model, the theoretical expressions were adjusted towards an improved agreement with the experimental results for the thermal behavior, but sacrificing the adherence with the flow results. The film thickness obtained through Suzuki et al. (1983) model are higher than those obtained experimentally, especially because they have neglected the entrainment effect, and it has already been demonstrated that the heat transfer coefficient increases with increased film thickness. Finally, according to Hagiwara et al. (1982), the experimental results here presented were obtained considering simultaneous development of the velocity and the temperature fields. The authors (Hagiwara et al., 1982) report that a fully developed region is achieved for channel lengths of around $\mathrm{Z} / \mathrm{D}=45$ in the cases considered. It is however a well-know fact that the heat transfer coefficients for simultaneous development of the velocity and the temperature fields are higher than those obtained for a fully hydrodynamically developed situation, as shown in Hewitt(1993).

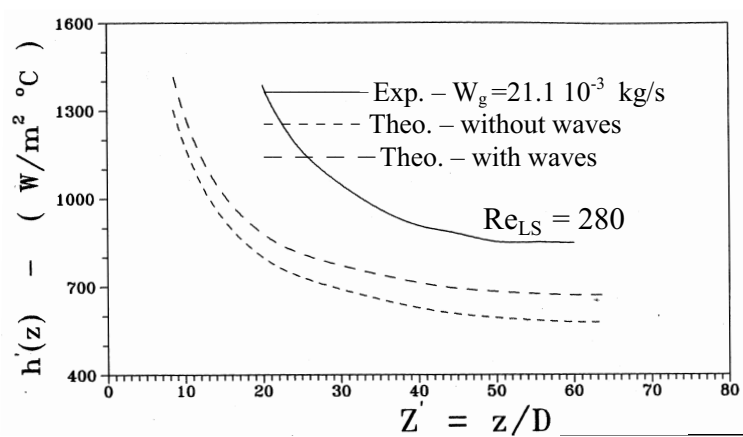

Figure 3. Comparison of theoretical (with and without interface waves effect) and experimental (Hagiwara et al., 1982) results for the heat transfer coefficient.

Figures 3 and 4 show a few comparisons of the present theoretical model for two-phase flow with interface waves effects, without entrainment and mass transfer effects, against available experimental results at different Reynolds number and gas flow rates. The interface waves effects are seen to be of relevance in the evaluation of the temperature field, as illustrated in Figs. 3 and 4 for the heat transfer coefficient analysis. Hagiawara et al. (1982) experimentally observed this aspect and they demonstrated that a significant portion of heat transfer enhancement in annular flow was due to the presence of interface waves, with a direct relation with their main characteristics, i.e., height, frequency and pattern. Figs. 3 and 4 confirm this behavior, in terms of the heat transfer coefficient along the channel length, for different values of gas flow rate and Reynolds number. It should be noticed that the interface waves effect is more significant at larger values of the Reynolds number associated with the liquid film.

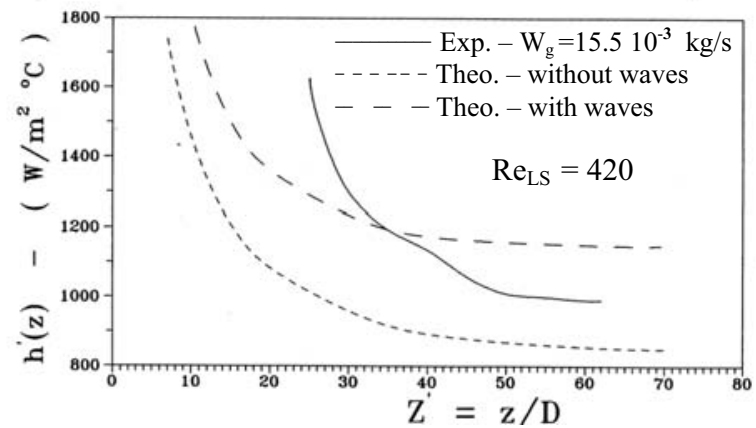

Figure 4. Comparison of theoretical (with and without interface waves effect) and experimental (Hagiwara et al., 1982) results for the heat transfer coefficient.

The results presented in Figure 5 were obtained considering flow with interface waves effects, without entrainment and mass transfer effects. Figure 5 demonstrates 
that not considering the waves effects results in a significant resistance to heat transfer. It has been demonstrated that introduction of the waves effect in the model allows an improved agreement with experimental results.

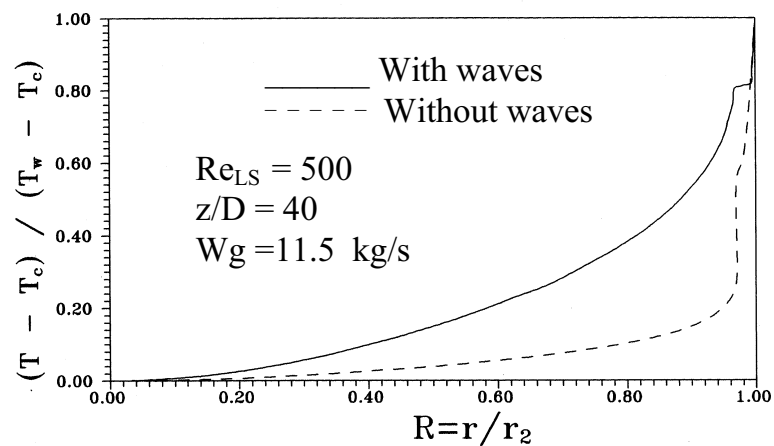

Figure 5. Influence of interface waves on temperature distributions in both phases.

\section{CONCLUSIONS}

In this paper is presented the analytical solution for a theoretical model in the representation of thermally developing forced convection in gas-liquid annular flow, utilizing the integral transform technique. Critical comparisons with few experimental results available indicate that some modeling extensions might be feasible under the same analytical framework, in order to improve the model adherence to the experimental findings.

We may also conclude that the wave effects in the interface are very important and heat transfer increases noticeably since the thermal resistance between the two phases is reduced. It is our understanding that a refined thermal modeling, for really predictive simulations, will be achieved once these aspects are more accurately accounted for within the interface energy balance.

\section{REFERENCES}

Barbosa J. R., Govan, A. H. and Hewitt, G. P., 2001, 'Visualisation and Modeling Studies of Churn Flow in a Vertical Pipe", Int. J. Multiphase Flow, Vol. 27, no. 12, pp. 2105-2127.

Collier, J. G., 1972, “ Convective Boiling and Condensation", McGraw-Hill, Second Edition.

Cotta, R.M., 1993, "Integral Transforms in Computational Heat and Fluid Flow", CRC Press, FL.

Cotta, R.M. and Nogueira, E., 1988, "On the Eigenvalues Basic to Diffusion Through Composite Media", Computational and Appl. Math., V. 7, pp. 201213.

Deissler, R.G., 1950, “Analytical and Experimental Investigation of Adiabatic Turbulent Flow in Smooth Tubes", NACA TN 2138.

Dobran, F., 1985, "Heat Transfer in an Annular TwoPhase Flow", J. Heat Transfer, Vol. 107, pp. 472-476.

Gill, L. E., Hewitt, G. F., Hitchon, J. W. and Lacey, P. M. C., 1963, "Sampling Probe Studies of the Gas Core in Annular Two-Phase Flow I- The Effect of Length on Phase and Velocity Distribution", Chem. Eng. Sci., Vol. 18, pp. 525-535.

Gill, L. E., Hewitt, G. F. and Lacey, P. M. C., 1964, "Sampling Probe Studies of the Gas Core in Annular Two-Phase Flow II- Studies of the Effect of Flow Rates on Phase and Velocity Distribution', Chem. Eng. Sci., Vol. 19, pp. 665-682.

Hagiwara, Y., Suzuki, K. and Sato, T., 1982, “ Studies on Thin Liquid Film of Annular - Mist Two-Phase Fhow. I - Wave Characterization and Heat Transfer', Mem. Faculty of Eng., Kyoto University, Vol. 44, pp. 309-328

Hewitt, G.F., 1993, "Fluid Dynamics and Heat Transfer in Annular Two-Phase Flow", Proc. of the $6^{\text {th }}$ Int. Symp. on Transport Phenomena in Thermal Engineering, Seoul, Korea.

Hewitt, G.F., Hall-Taylor, N. S., 1970, “Annular Two Phase Flow", Chemical Engineering Division, A.E.R.E., Harwell, England, Pergamon Press, Oxford.
Jensen, M. K., 1987, "The Liquid Film and the Core Region Velocity Profiles in Annular Two-Phase Flow", Int. J. Multiphase Flow, Vol. 13, No. 5, pp. 615-628.

Kays, W.M. and Crawford, M.E., 1980, "Convective Heat and Mass Transfer", $2^{\text {nd }}$ Edition, McGraw-Hill, New York.

Mikhailov, M.D. and Özisik, M. N., 1984, "Unified Analysis and Solutions of Heat and Mass Diffusion", John Wiley, New York.

Nogueira, E., Brum, N.C.L. and Cotta, R.M., 1990, “Annular Gas-Liquid Flow in Vertical Ducts with Liquid Entrainment in the Core", Proc. of the $3^{\text {rd }}$ National Thermal Sciences Meeting, ENCIT90, Vol. 1, pp. 559-564, Itapema, SC, Brazil.

Nogueira, E., 1993, "Solução Analítica para Escoamento e Transferência de Calor em Regime Anular Vertical", Tese de doutorado, PEM/COPPE/UFRJ, Brasil.

Nogueira, E., Cotta, R.M., Brum, N.C.L. and Kakaç, S., 1995, "Analytical Solution with Algebraic Turbulence Models for TwoPhase Gas-Liquid Annular Flow \& Heat Transfer", Two-Phase Flow and Experimentation, Rome, Italy, October 9-11, Vol. 2, pp. 289-396.

Nogueira, E., Cotta, R.M., Brum, N.C.L., and Kakaç, S., 1996, "Integral Transform Solution of Heat Transfer in TwoPhase Gas-Liquid Annular Flow", Int. J. Heat \& Technology, V.14, no.1, pp.97-120.

Silvestri, M., 1964, "Fluid Mechanics and Heat Transfer of Two-Phase Annular Dispersed Flow", Adv. Heat Transfer, Vol. 1.

Suzuki, K., Hagiwara, Y. and Sato, T., 1983, "Heat Transfer and Flow Characteristic of Two-Component Annular Flow", Int. J. Heat \& Mass Transfer, Vol. 26, No. 4, pp. 597-605.

Zabaras, G., Dukler, A. E. and Maron, M. D., 1986, "Vertical Upward Cocurrent Gas-Liquid Annular Flow", AIChE J., Vol. 32 , No. 5 , pp. $829-834$

Walley, P.B., 1987, 'Boiling, Condensation and Gas-Liquid Flow", Clarendon Press, Oxford, UK.

Wolf, A., Jayanti, S., and Hewitt, G. P., 2001, "Flow Development in Vertical Annular Flow", Chemical Engineering Science, Vol. 56, No. 10, pp. 3221-3235. 